\title{
A comment on the paper 'Biomarkers in Multiple Sclerosis: a review of diagnostic and prognostic factors' by Sapko et al.
}

\author{
Klaudia Sapko, Anna Jamroz-Wiśniewska, Michał Marciniec, Marcin Kulczyński, \\ Anna Szczepańska-Szerej, Konrad Rejdak
}

Chair and Department of Neurology, Medical University of Lublin, Poland

(Neurol Neurochir Pol 2021; 55 (1): 117)

\section{To the Editors:}

Firstly, we would like to thank you for the opportunity to respond to the issues raised in Prof. Lewczuk et al's Letter to the Editors [1] and to clarify the aim of our paper [2].

We would also like to thank Lewczuk et al. for their interest in our paper and for taking time to express their comments [1]. We acknowledge the importance of cerebrospinal fluid (CSF) biomarkers in multiple sclerosis (MS), especially oligoclonal bands. In their Letter, the authors explain in detail oligoclonal bands' methodology and note concerns about an insufficient approach to MS biomarkers' methodology in our paper.

However, the primary objective of our review was to focus on prospective and future biomarkers in MS not, due to limited space, to discuss in detail currently available biomarkers that have been already reviewed previously [3-6]. Therefore, we thank Lewczuk et al. for their valuable comments. These will be an additional resource for readers interested in MS biomarkers.

Ultimately, we agree with the authors that continued research into MS biomarkers is needed to fully understand the pathogenesis of this disease and improve diagnostics.

\section{References}

1. Lewczuk P, Kornhuber J, Mroczko BA, et al. A comment on the paper 'Biomarkers in Multiple Sclerosis' by Sapko et al. Neurol Neurochir Pol. 2021; 55 (1): 115-116, doi: 10.5603/PJNNS.a2020.0074, indexed in Pubmed: 33026646.

2. Sapko K, Jamroz-Wiśniewska A, Marciniec M, et al. Biomarkers in Multiple Sclerosis: a review of diagnostic and prognostic factors. Neurol Neurochir Pol. 2020; 54(3): 252-258, doi: 10.5603/PJNNS. a2020.0037, indexed in Pubmed: 32462652.

3. Link H, Huang YM. Oligoclonal bands in multiple sclerosis cerebrospinal fluid: an update on methodology and clinical usefulness. J Neuroimmunol. 2006; 180(1-2): 17-28, doi: 10.1016/j.jneuroim.2006.07.006, indexed in Pubmed: 16945427.

4. Gastaldi M, Zardini E, Leante R, et al. Cerebrospinal fluid analysis and the determination of oligoclonal bands. Neurol Sci. 2017; 38(Suppl 2): 217 224, doi: 10.1007/s10072-017-3034-2, indexed in Pubmed: 29030765.

5. Pryce G, Baker D. Oligoclonal bands in multiple sclerosis; Functional significance and therapeutic implications. Does the specificity matter? Mult Scler Relat Disord. 2018; 25: 131-137, doi: 10.1016/j. msard.2018.07.030, indexed in Pubmed: 30071507.

6. Lo Sasso B, Agnello L, Bivona G, et al. Cerebrospinal Fluid Analysis in Multiple Sclerosis Diagnosis: An Update. Medicina (Kaunas). 2019; 55(6), doi: 10.3390/medicina55060245, indexed in Pubmed: 31167509. 